\title{
Virtual Flipped Class and Laboratories for Medical Electronics Course
}

\author{
JEAN-Michel I. MAAREK \\ Department of Biomedical Engineering, University of Southern California, 1042 Downey Way, Los Angeles, CA 90089, USA
}

(Received 19 June 2020; accepted 13 October 2020; published online 3 November 2020)

\begin{abstract}
This paper describes the adaptation of a flipped Biomedical Electronics course with laboratories to remote learning at the start of the Covid-19 pandemic. In class collaborative work on problem sets was replaced by group work (4-5 students) in Zoom breakout sessions. When the groups assembled at random for each class had sufficiently progressed on a problem, a detailed solution was typed on the Multisim circuit simulator desktop (National Instruments) shared on the instructor screen. A laboratory project dealing with the development of an electromyograph (EMG) was redesigned for in-depth exploration of each circuit block composing the EMG circuit that was only feasible with the circuit simulator. The students progressed through the remote section of the course at the same rate as they had in the physical classroom in prior years. Student pairs finished a more complete virtual EMG laboratory project without being hampered by manipulation errors that are typical of novices when assembling hardware circuits. We conclude that a flipped biomedical electronics course can successfully be offered remotely and that virtual electronics laboratories that make use of a circuit simulator can provide a complete and meaningful learning experience.
\end{abstract}

Keywords-Remote learning, Flipped classroom, Electronics laboratories, Circuit simulator.

\section{BACKGROUND}

The Medical Electronics course at our institution is organized as a flipped class (two 80-min sessions/week) with design-based laboratories (one 3-h lab/ week-morning or afternoon times) in which junior and senior students analyze, design, assemble, and test prototypes of medical instruments. ${ }^{14,15}$ Online video lessons posted on the learning management system (LMS) Blackboard present methods of analysis and design of fundamental electronic circuits encountered in these instruments, e.g. amplifiers assembled with operational amplifiers, active filters, comparators, diode circuits, and transistor switches. In class, students practice in small groups analyzing variants of these circuits.

The labs are mainly devoted to two multi-week projects (dual-supply electrocardiograph amplifier and single-supply electromyograph amplifier) divided into self-contained functional blocks which are based on circuits studied in class. Students design and implement a functional block each week to satisfy specified requirements and use as reference documentation application notes and professional tutorials. ${ }^{11,5,16}$ Preparation for the labs includes developing and testing a model of each circuit block with the simulation software Multisim (National Instruments). In lab, student pairs assemble and test the circuit block on a breadboard and compare its experimental performance with design requirements and predictions of the simulation model. Students spend a considerable fraction of each lab identifying and correcting manipulation errors: using a component with the wrong value or the wrong component, wiring errors, etc. Locating these errors is complicated by sloppy wiring habits, ${ }^{10}$ which take considerable time to remedy in spite of a tutorial all students study before the first lab and repeated recommendations from the instructor and teaching assistants. 


\section{CHALLENGE STATEMENT}

The challenge was to reorganize the last six weeks of the course for remote operation while keeping the essential features and processes described above which the students had become accustomed to during the semester's first nine weeks.

\section{Novel Initiative}

Addressing the challenge required reorganizing the classroom activities so they could be attended remotely and reformatting the labs such that students could design and validate their circuits exclusively with the Multisim circuit simulator (online appendix). All Zoom meetings were scheduled at the regular classroom and laboratory times.

Ahead of each remote class, problem sets were posted on the LMS both as a static document and a Multisim file which comprised the circuits the students would analyze together. Class started with a 5-min summary of the video lesson followed by a brief question-and-answer period. Thereafter, students were randomly grouped into Zoom breakout sessions (4-5 students/session) to work on each problem. The instructor and teaching assistant roamed the breakout sessions to encourage student participation and assess progress. Groups "raised hand" to call for help. This approach emulated the operation of the physical classroom such that after a short adaptation, class progressed at the rate it had in previous years. Once most groups had made significant progress, the students were reunited in the main session. Steps to reach the solution and the numerical answers were typed as comments on the instructor's Multisim desktop with participation from the students. Pencil-and-paper answers were augmented with estimations obtained with Multisim's simulation tools (voltage and current probes, oscilloscope, etc.) which allowed the instructor to point out differences between simple models used to solve the problems by hand and more complex models used in the simulator. Multisim was also used to validate the solutions of design problems. For instance, student groups designed active filters by hand using established algorithms ${ }^{12}$ and chose component values within certain tolerance ranges. With the whole class, the frequency response of the filter was simulated using students' values and the response's characteristics (critical frequency, roll-off rate, passband gain) were compared to the design requirements. After each class, the solutions and a link to the Zoom recording of the session were posted on the LMS for review.

The transition to remote learning occurred just prior to starting the electromyograph (EMG) amplifier project which the students develop from documenta- tion presented in instructor-selected references. ${ }^{5,16}$ The assignment was restructured to design the circuit in 5 functional blocks (Table 1). Each week, the students studied material from the references and parts data sheets to prepare for designing and testing one functional block in the virtual lab. The functional blocks included design novelties made possible by the circuit simulator (weeks 2 and 3) or circuitry that was not part of the original EMG circuit built in hardware (weeks 4 and 5). These innovations were made possible by the richness of components available in Multisim and the speed at which students tested and validated their circuits in the absence of manipulation errors. ${ }^{10}$ Hardware comparators in week 4 to detect the EMG signal reaching pre-defined intensity levels replaced software detection of these thresholds with a microcontroller in the physical circuit, as this was difficult for the students to implement in Multisim.

Students completed multiple validation tests of their simulated circuits which would have been too timeconsuming or impossible to achieve in a hardware circuit (Table 1). In week 2, they used Monte-Carlo simulations to vary randomly resistor and capacitor values within their tolerance range and visualize the effect of parts tolerance on the frequency responses of the active filters. In week 3, they examined the effect of part tolerance on the behavior of a precision rectifier circuit. Students also compared the frequency responses of a variable gain amplifier for four gain settings and related their observations to the nominal gain-bandwidth product of the operational amplifier chip. Functional block 5 (opto-isolation of the amplifier front-end) was added because the students had worked faster to complete the first four parts, and it allowed them to explore electrical protection in bioelectric systems.

During the laboratory Zoom sessions, after a brief overview of the weekly task, students assembled in breakout sessions with the lab partner who they had worked with prior to moving online. Student pairs could choose to attend the morning or the afternoon session to better fit their schedule and geographic location. Student pairs called for help and shared their screens as needed. All student teams completed the entire project, which seldom happened in previous years in the physical labs.

\section{Reflection}

The main differences between the online classroom sessions and the physical sessions included (1) random grouping the students for the activities, (2) seamless availability of a permanent record comprised of the problem solutions typed in the Multisim files and the 
TABLE 1. The single-supply electromyograph (EMG) design was divided in 5 functional blocks and the students developed one functional block each week, which they tested first individually and then connected to the previously-developed functional blocks.

Week 1 - functional block 1: 5V dc single-supply instrumentation amplifier and $2.5 \mathrm{~V}$ voltage referenceCircuit design novelty: none

$\mathrm{N} \# 1$ : Determine effect of input bias currents ( $\left.\mathrm{l}_{\text {bias }}\right)$ and input offset voltage $\left(\mathrm{V}_{\mathrm{os}}\right)$ on amplifier dc output voltage $\left(\mathrm{V}_{\text {out }}\right)$

$\mathrm{N}$ \#2: Experimental amplifier frequency response
$\mathrm{T}$ : Use 4 sets of ground return resistors at the amplifier's inputs and measure $\mathrm{V}_{\text {out }}$ for each set

T: use "ac sweep" to change input frequency and generate output response. Measure upper and lower critical frequencies $(-3 \mathrm{~dB}$ points)
$A$ : use resistor and $V_{\text {out }}$ values to compute $I_{\text {bias }}$ and $V_{\text {os. }}$. Compare to nominal values from amplifier data sheet

A: relate lower critical frequency to $\mathrm{R}-\mathrm{C}$ high pass filter at amplifier's inputs and upper critical frequency to gain-bandwidth product

Week 2 - functional block 2: signal conditioning with high-pass and low-pass filtersCircuit design novelty: design 4-pole Chebyshev low-pass filter with Webench filter software design tool (Texas Instruments)

$\mathrm{N} \# 1$ : visualize effect of com- T: "Monte-Carlo" simulation to generate 20 frequency ponent tolerances on filter frequency response

$\mathrm{N}$ \#2: measure time-domain delay through filters response curves for randomly selected passive components within tolerances $(10 \%$ resistors, $20 \%$ capacitors)

T: use "interactive simulation" oscilloscope to measure time difference between input and output waveforms at critical frequencies
A: measure highest and lowest values of critical frequencies. Compare with theoretical values determined from filter design

A: relate experimental delays to theoretical filter group delays estimated with Webench

Week 3 - functional block 3: Single-supply non-inverting op-amp amplifier, precision rectifier, EMG envelope circuitCircuit design novelty: design of bandwidth limited op-amp amplifier to extract EMG envelope

$\mathrm{N} \#$ 1: measure amplifier frequency response for different gain values

$\mathrm{N} \# 2$ : sensitivity of precision rectifier response to component values

$\mathrm{N}$ \#3: validation of EMG envelope detection circuit
$\mathrm{T}$ : use "ac sweep" to generate frequency response curve for 4 amplifier feedback resistors placed in circuit with a rotary switch

T: use "parameter sweep" to vary component values of precision rectifier circuit by $\pm 10 \%$ in 21 increments

$\mathrm{T}$ : input AM modulated voltage signal and measure with oscilloscope output waveform at carrier frequency and modulation frequency
A: determine dc gain and critical frequency for each resistor value. Relate to op-amp gain bandwidth product

A: determine dc operating point for each component value. Interpret results based on working principle of precision rectifier circuit

A: determine amplitude ratio of signals at carrier and modulation frequencies at the input and the output of the envelope detector

Week 4 - functional block 4: comparators with hysteresis to detect EMG crossing selected thresholds and energize transistor switchesCircuit design novelty: entire circuitry

N \# 1: design two comparators with hysteresis T: apply DC interactive voltage source at comto detect EMG amplitude crossing pre-selected intensity thresholds

\section{parator input and measure levels at which output} switches from "low" to "high"
A: relate "threshold" voltages to theoretical predictions from comparator design calculations

Week 5 - functional block 5: optocoupler circuit to isolate electrically EMG amplifier front endCircuit design novelty: entire circuitry

$\mathrm{N} \# 1$ : design and test voltage-to-current converter to control opto-coupler LED

$\mathrm{N} \# 2$ : characterize experimentally current transfer ratio (CTR) of opto-coupler component
T: apply DC interactive voltage source and measure LED current for different input voltages

$\mathrm{T}$ : measure opto-coupler photodiode current for different LED currents
A: tabulate LED current output and relate to component values of voltage-to-current converter

A: determine ratio photodiode current / LED current and relate to nominal CTR from datasheet

The virtual circuit design novelty in comparison to the hardware-based EMG project of previous years is indicated below the block description. "N \# i" $(1 \leq \mathrm{i} \leq 3)$ designate the new virtual experiments the students developed to validate the functional block. "T" describes to the matching test the students performed using Multisim's virtual measurement instruments. "A" refers to the analysis the students completed on the test data.

Zoom recordings, and (3) augmenting the circuit analyses using the capabilities of Multisim.

Random grouping of the students ensured that on average, students with different ability levels worked together, which was different from patterns observed in the physical classroom. In the latter, juniors and seniors usually worked in separate groups. The stronger students tended to assemble together, resulting in groups of weaker students. This pattern has been previously reported for cooperative learning groups. ${ }^{7}$ Student groups with heterogeneous levels of ability have been found to be more successful in terms of performance and student satisfaction. ${ }^{7,6}$ Low-ability students perform better when included in heteroge- 
neous groups ${ }^{13}$ which also benefit high-ability students as they practice verbalizing in different ways their approach to solving the problem sets. New student groups were formed at random for each class in this first attempt. It has been recommended to keep stable groups for a month or longer to build group cohesiveness and student interdependence. ${ }^{7}$ For future offerings, whether online or in person, instructorformed heterogeneous student groups will be used and groups will be reshuffled at most a few times in the semester.

Producing a record of all classroom activities (breakout sessions excluded) was effortless and benefited students who could not attend class synchronously. Recording all classroom activities may be difficult to implement in the physical setting without a special infrastructure. However, typing the main steps to reach the problems' solutions on a permanent record with student input would be easy to implement and preferable to the ephemeral solution written on the classroom whiteboard or to posting instructor's solutions prepared without student input. Systematic use of the circuit simulator opened new possibilities for computation and visualization which were implemented without breaking the flow of the classroom activities.

In the virtual labs, students took advantage of the capabilities of Multisim to explore empirically practical implementation issues in electronic circuits which are seldom investigated in hardware labs. These included examining the effects of parts tolerance on circuit behavior and the differences between realistic component models and their idealized counterparts. Circuit simulators have been found to be effective tools for teaching electronics and circuit analysis. ${ }^{10,3}$ Thus, to train students in debugging circuits, Hodge et al. ${ }^{10}$ developed a virtual environment in which circuits included random wiring errors and components with erroneous values. The students used virtual test instruments to analyze the circuit behavior and identify the faults. Campbell and colleagues ${ }^{3}$ investigated students who trained exclusively in physical electronics labs with students who trained in matched simulated labs $(80 \%$ time $)+$ physical labs $(20 \%$ time $)$. Student performance was assessed with a written test covering lab theory and skills and by measuring the amount of time needed to complete a new physical lab. Students who had trained in the hybrid format performed better on the written exam than those who only trained in the physical labs. Both groups took the same amount of time to complete the new lab, suggesting lab skills had at a minimum not been adversely affected by training mostly in the virtual environment. Hall ${ }^{9}$ divided freshman-level and junior-level electronic technology students at random to complete half of their electronics labs in a simulated environment and the other half using hardware. Post-lab testing demonstrated that the two groups learned the concepts presented in the labs equally well. Analysis of the students' perceptions of their learning gains revealed that the freshmen felt they learned as much in either context with respect to theory and to troubleshooting skills. The juniors who had prior experience in physical labs thought they learned more in the latter setting and preferred it over the virtual labs. We surmise that students can learn equally well electronics in virtual labs and physical labs but that they learn different things. The simulated labs enable students to explore more deeply the operation of a circuit and issues of importance to engineers and designers such as the effect of parts tolerance on circuit performance. Hands-on troubleshooting skills that are useful for laboratory practice are better acquired in physical labs. When physical labs are not possible, many benefits can still be derived from simulated electronics laboratory experiments. Simulated experiments have been found to be useful and practical to teach other subjects relevant to biomedical engineering, including chemical reactor processes ${ }^{17}$ and human pathophysiology. ${ }^{2}$ Simulated experimental environments take time and resources to develop. We had the advantage that Multisim was already used in our course, and that, like other SPICE-based simulators, Multisim is relatively inexpensive and widely available.

The simulated EMG project included an additional functional block as compared to the hardware project used in previous years. Yet, all students completed the entire project, which rarely happened in the past. Assembling a hardware circuit from a schematic involves several cognitive tasks beyond validating the circuit design. Components symbols and pin locations must be converted into their physical counterparts. Component values must be translated from letters and numbers to colored bands (resistors) and inscriptions on integrated circuits. Connections on the schematic must be matched to holes on a solderless breadboard. Cognitive load theory asserts that concurrent tasks overload working memory in novices and slow down or reduce learning. ${ }^{4,1}$ The theory distinguishes between germane load which helps learning and extraneous load which makes learning more difficult or impossible. By removing the heavy cognitive effort associated with assembling the hardware, the simulated labs likely reduced cognitive load and enabled the students to work faster and complete an expanded version of the project from the design standpoint. Additional explorations of the circuit properties became possible. When the main cognitive task is to learn to assemble a hardware circuit, we suggest that cognitive load can be reduced by using software that displays a graphical representation of the parts wired on the breadboard, 
such as Fritzing ${ }^{8}$ or the simulated breadboard of Multisim. Clear identification of the expected learning gains from laboratories can help define the germane load and result in deeper and more satisfying learning experiences for the students.

The course content covered in the six weeks of online learning was equivalent to that covered in the same time period in the physical classroom in prior years. This observation suggests that switching to online learning did not diminish the amount of course material that could be taught and learned. The course learning objectives include: (1) Describe electrical characteristics of transducers, diodes, integrated amplifiers, and bipolar junction transistors; (2) Analyze and design circuits built with these electronic components; (3) Design, implement, and validate electronic circuits using these components with laboratory tools; (4) Utilize a circuit simulation software to analyze and design electronic circuits.

Achievement of the first two objectives is assessed with written examinations (5-7 short quizzes, a midterm exam, and a comprehensive final exam) and can be accomplished in the same manner for content covered in the classroom or remotely. Each examination comprises objective questions that test the students on a learning outcome from one of 13 lessons covered in the semester by having the students solve a short problem and identify the correct answer among 5 choices. Examples of lesson learning outcomes include (1) determine the effects of the input offset voltage and input bias current of a "real" op-amp on the DC output voltage of an op-amp amplifier, (2) design Sallen-Key second-order low-pass and high-pass filters using filter tables, and (3) analyze and design inverting and non-inverting comparators with hysteresis. In the semester, about 80 objective questions are used to directly assess the students on the detailed lesson learning outcomes which in turn reflect the more global course learning objectives.

Course learning objectives (3) and (4) are assessed from the preparatory documents and laboratory reports submitted by the students prior to and after each laboratory. Each laboratory assignment lists several deliverables for the students to include in their reports. Examples include a wiring diagram with component values for a 4-pole low pass filter designed to achieve a specified critical frequency and response, and the analysis of the filter characteristics, including the experimental frequency response curve, critical frequency, and roll-off rate. The students' reports are assessed with checklists that assign points to each deliverable listed in the assignment. While the design, implementation, and validation testing are different for simulated circuits and hardware-based circuits, comparable methods and tools are used for the design and testing, which the students should highlight in the deliverables they turn in. In the online laboratories, students learned in greater depth the use of the circuit simulation software Multisim which would be reflected in the list of expected deliverables included in the laboratory assignments and in the students' writeups.

In conclusion, the flipped class structure of our Medical Electronics course facilitated content delivery by means of virtual collaboration tools. Classroom activities completed online emulated the approach used in the physical classroom. Virtual labs took advantage of the capabilities of the circuit simulation software Multisim to enable students to experiment and investigate features of a complex electronics project that would have been difficult to undertake in physical labs.

\section{ELECTRONIC SUPPLEMENTARY MATERIAL}

The online version of this article (doi:https://doi.or $\mathrm{g} / 10.1007 / \mathrm{s} 43683-020-00037-6)$ contains supplementary material, which is available to authorized users.

\section{ACKNOWLEDGEMENTS}

The contributions of the two teaching assistants, Ms. Chang Liu and Mr. Jeff Tanedo, to the smooth operation of this course is gratefully acknowledged.

\section{REFERENCES}

\footnotetext{
${ }^{1}$ Abeysekera L, Dawson P. Motivation and cognitive load in the flipped classroom: definition, rationale and a call for research. High Educ Res Dev. 2015;34:1-14.

${ }^{2}$ Bintley HL, Bell A, Ashworth R. Remember to breathe: teaching respiratory physiology in a clinical context using simulation. Adv Physiol Educ. 2019;43:76-81. https://doi. org/10.1152/advan.00148.2018.

${ }^{3}$ Campbell JO, Bourne JR, Mosterman PJ, Brodersen AJ. The effectiveness of learning simulations for electronic laboratories. J Eng Educ. 2002;91:81-7. https://doi.org/10. 1002/j.2168-9830.2002.tb00675.x.

${ }^{4}$ Clark R, Nguyen F, Sweller J. Efficiency in learning evidence-based guidelines to manage cognitive load. San Francisco, CA: Pfeiffer; 2006.

${ }^{5}$ Colliard-Piraud S. Application note AN 4995 - using an electromyogram technique to detect muscle activity. ST MicroElectronics; 2017.

${ }^{6}$ Felder R, Brent R. Effective strategies for cooperative learning. J Cooper Collabor Coll Teach. 2001;10:69-75.

${ }^{7}$ Felder R, Woods D, Stice J, Rugarcia A. The future of engineering education II. Teaching methods that work. Chem Eng Educ. 2000;34:26-39.
} 
${ }^{8}$ Fritzing. https://fritzing.org/.

${ }^{9}$ Hall Jr TM. Using simulation software for electronics engineering technology laboratory instruction. St Louis, MO.

${ }^{10}$ Hodge H, Hinton HS, Lightner M. Virtual circuit laboratory. J Eng Educ. 2001;90:507-11. https://doi.org/10.1002/ j.2168-9830.2001.tb00632.x.

${ }^{11}$ Kitchin C, Counts L. A designer's guide to instrumentation amplifiers. 2nd ed. Wilmington, MA: Analog Devices; 2004.

${ }^{12}$ Kugelstadt T. Active filters design techniques, excerpted from Op Amps for everyone. Texas Instruments - Literature number SLOA088.

${ }^{13}$ Lou Y, Abrami PC, Spence JC. Effects of within-class grouping on student achievement: an exploratory model. J Educ Res. 2000;94:101-12. https://doi.org/10.1080/002206 70009598748.
${ }^{14}$ Maarek JMI. Student feedback in inquiry-based laboratories for Medical Electronics course. In: Proceedings of ASEE. Columbus, Ohio; 2017.

${ }^{15}$ Maarek JMI, Kay B. Assessment of performance and student feedback in the flipped classroom. In: Proceedings of ASEE. Seattle, WA; 2015.

${ }^{16}$ Mozhanova M. Design of a high-resolution surface electromyogram (EMG) conditioning circuit. Worcester Polytechnic Institute: Qualifying Project Report; 2012.

${ }^{17}$ Wiesner TF, Lan W. Comparison of student learning in physical and simulated unit operations experiments. J Eng Educ. 2004;93:195-204. https://doi.org/10.1002/j.2168-983 0.2004.tb00806.x.

Publisher's Note Springer Nature remains neutral with regard to jurisdictional claims in published maps and institutional affiliations. 